\title{
Immersive Energy Network Operation System for Renewable Resources
}

\author{
Amr Elsaadany and Mohamed Soliman \\ Department of Computer Engineering,Pharos University in Alexandria
}

\begin{abstract}
: with the proliferation of scattered new energy sources, there becomes a need to manage this growing phenomenon. The tracking gives a comprehensive view of the availability of the energy sources and aids in the usability of the produced energy. In this paper, we propose a new network operation center conceptthat provides technical support for the renewable energy sources. First, a comprehensive database of all the vital information of each energy site is designed. Second, the infrastructure of the network operation center is proposed with all the requirements needed to support the monitoring and control of the energy sites remotely. Third, an immersive virtual environment is usedto support the simulation and management of the renewable energy production. The 3D virtual environment can be used in both management and training for the technical staff and to stimulate new operational scenarios.
\end{abstract}

Keywords: renewable energy; network operation center; network connectivity; operational scenarios; immersive environment

\section{Introduction}

As new energy sources are beginning to spread in developing countries, there is a need to track and monitor them. Moreover, there is a need to control the operation of these renewable energy resources. A monitoring and control system can range from simple on-site computerized maintenance terminal to a full Network Operation Center (NOC) on the scale of city or even the whole country. The NOC will be connected to energy source controllers via some network connection. The controllers will then connect to the sensors in the power system and convert the digital data to sensor signals and vice-versa. A data network is used to connect the on-site controllers with the control centers. The communication can be based on wired or wireless media. Examples include leased telephone lines, WAN circuits, licensed and unlicensed radio, cellular, microwave, or even satellite for remote areas that have no network coverage.

An immersive virtual environment, represented by a practical virtual world implementation, is incorporated. Through the virtual world, it is possible to add visualization combined with other virtual world abilities such as avatar collaborative interaction [1]. The benefits and potential of adding an immersive environment is shown in relation to state of the art and how it can contribute to increased awareness and education (for example by visualizing energy production). The awareness of the end-user about energy production and the possible operational scenarios leads to energy saving practices.One of the main purposes of this work is to come up with a computerized tracking, monitoring and control system for the renewable energy sources. The system contains a database of the available energy sources and their capacity data. The system is capable of providing information about the usability and availability of the energy sources.

This paper is organized as follows. Section 2 provides introductory background on network operation centers. Section 3 describes the needs for the new system along with its requirements. Section 4 discusses the use of immersive environment as a candidate for NOC by giving the requirements and proposing a conceptual view of the integration of the concepts. Section 5 details maintenance scenarios that are realizable in an immersive environment that serves the NOC concept. Section 6 concludes and provides future work and limitations.

\section{NOC Background}

This section provides the needed background on NOC and their main functionalities.NOC is a centralized location where IT technicians can directly support the efforts of remote monitoring and management of network elements.The NOC staff is responsible for monitoringthe network element and make decisions and adjustments to ensure optimal network performance [2]. The NOC staff can create alerts that identify and categorize network element issue based on severity, type, and a number of other criteria items, when any action is required.NOC users have other capabilities such as application software installations, troubleshooting, and updating. They must have electronic data management as well as backup and storage management services. They need tools to perform network discovery and assessments, policy enforcement, firewall and intrusion prevention as well asscanning and remediation. They should be able to provide optimization and quality of service reporting and performance reporting and improvement recommendations. 
The NOC technical users are categorized based on the job levels, which indicate expertise and problemsolving ability. A first level user deals with hardware failure alerts while senior level user may be assigned with the problems beyond just the failed hardware and goes into system analysis. The NOC need not be owned by the entity producing the energy; instead other company can own the NOC system. These NOC companies specialize in managing IT services and are called Managed Service Providers (MSPs). Those companies can manage multiple clients at the same time from the same NOC site [3].The proposed Energy NOC (ENOC) is based on the outsourcing concept where a third party organization performs the network operations. This saves the need for fully qualified staff to maintain the network operations center and saves the fixed infrastructure costs. Through outsourcing, a NOC's technical teams can act as an extension of the existing teams in order to ensure the uptime and the satisfaction for all the customers. Within the NOC itself, a typical wall unit is usually used to provide overall view of the network components. See Figure 1 for an example of such wall unit [4].

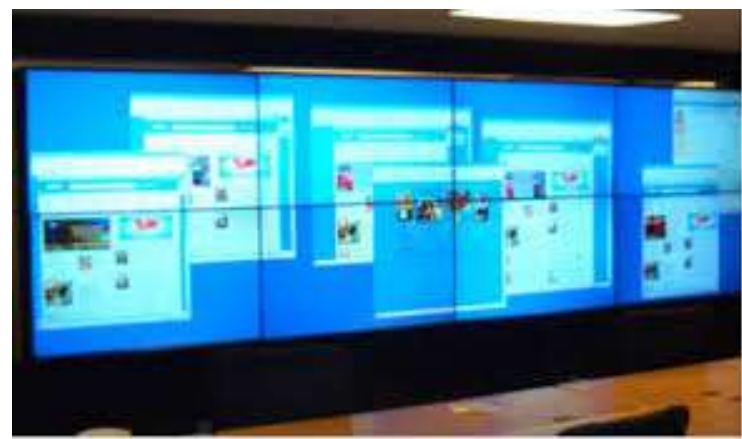

Fig. 1 Example of a NOC wall unit [4]

\section{NOC Requirements}

In addition to furnishing and staffing the NOC, procedures must be put in place for the proper operation of the network. The network element must be tracked using a comprehensive database of all the components. The NOC operations should be available $24 / 7$ in order to provide continued support.It is very important to notice the energy consumption of the NOC itself. Energy aware network operations are presented in [5], where energy saving approaches are considered. It is found that NOC energy saving can be obtained by appropriately adjusting the active network elementsamong other energy saving techniques.

Aclosely related topic is the wise energy utilization by consumers and usage of smart meters in this regard. Smart home energy related activities are discussed in [6] whichdescribe the smart home energy management system and the challenges associated with its energy management and the possible solutions. The use of smart meters increases the consumers' awareness of their energy usage based on real-time measurements.Accessing the installed energy site is a main NOC requirement which allows the technicians to monitor and control the equipment. Rbee Solar is a web application that allows the monitoring the photovoltaic (PV) installations [7]. The monitoring products are equipped with a GSM/GPRS modem and a built-in and preactivated SIM card. The device screen indicates the signal strength and provides reliable, autonomous and independent data transmission.

A web monitoring system for solar power is proposed in [8]. Solar monitoring is very important especially for installations that have large number of solar panels. Monitoring allows early detection if solar power output falls below expected level which can result from one or more of the solar panels goes down. The web-enabled software can monitor and control the solar panel by using an internet connection. Figure 2 shows the general structure of solar power web monitoring systems. The data logger device reads data received (wirelessly) from the solar panels and stores information in a storage device to be read by the web server through the internet.

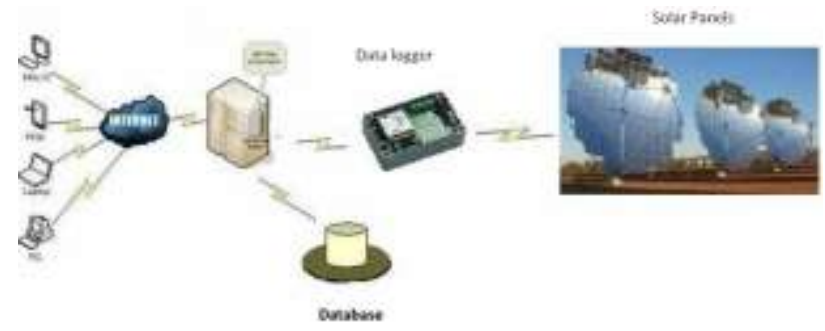

Fig. 2General Structure of Solar Power Web Monitoring System [8]

Netbiter is a system that can keep track of all remote renewable energy installations [9]. Solar power generators can be monitored online in order to check the mainoperationalparameters such as temperatures and 
power output. The system can generate statistical reports and more importantly, it can generate alarms to detect if some parameters reach certain levels. The alarms are sent by the system to the NOC staff via SMS or email while managing mobile fleets through GPS functionality. The Netbiter communication gateway connects to the solar panel using a serial, Ethernet, or I/O connection as shown in Figure 3. The gateway sends information via the internet or the cellular network, to the NOC.

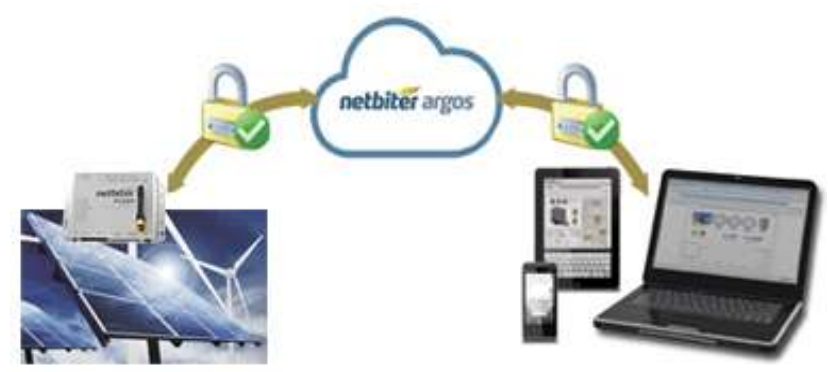

Fig. 3Netbiter Communication System [9]

The control of various parameters in the solar plant can be done via Supervisory Control and Data Acquisition (SCADA) network [10]. SCADA operates using signals over communication channels to provide control of remote equipment covering multiple sites and large distances. The Programmable Logic Controllers (PLCs) connect to sensors and convert sensor signals to digital data. The data is then transmitted to the control center via wired media such as leased telephone lines and WAN circuits or via wireless media such as licensed/unlicensed radio, cellular, and satellite.Therefore, the data from the solar panels can be received wirelessly or using a serial, Ethernet, or I/O connection. On the equipment panel side, a connection point capable of extracting and sending the data all the way to the NOC, is needed. The data, in turn, are stored and processed for the purpose of monitoring and controlling the energy source.

With the increasing penetration of wind energy, the wind plants should be capable of providing power tracking and control [11]. Grid operators must maintain the necessary balance between power generation and load, which in turn regulates the grid frequency. The grid frequency is related to the total power generated and the power consumed by system loads; if the power generation exceeds the load, then the grid frequency will go up. Wind turbines can be controlled by the Active Power Control (APC) unit which is responsible for varying the active power output of the turbines in order to compensate for power fluctuations or unplanned events such as sudden loss of power generation. Figure 4 shows an example of a standard interconnection of a wind farm controller with the grid operator, the utility grid, and the individual turbines.

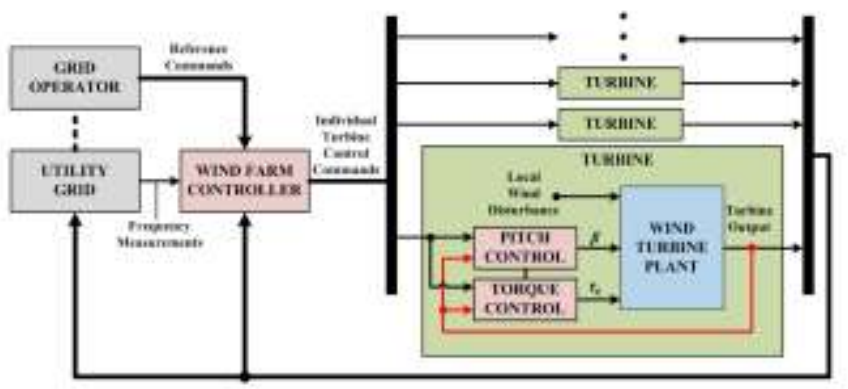

Fig. 4 Example of a wind plant controller [11]

The NOC should allow its users to log on, in order to check the operational parameters of the energy source e.g., solar equipment, via a computer, tablet or smart phone. The ability to check system parameters remotely and the ability to get system statisticsreduce expensive service visits by the technicians. To support the NOC functionalities, all the devices that are monitored and controlled must be defined to the NOC users. A database containing all the managed elements is thus required to store all the needed information and the relations between the various entities. For an energy source, attributes such as the device type, GIS location, maintenance schedule, and data interface type, site name and ID, etc. should be included in the database. For the NOC entity, user types must be specifiedasthey have different job requirements; NOC engineer, field engineer, or NOC manager.

A simplified version of the NOC database is shown in Figure 5, whereas a much more detailed version is used in the actual design. For instance, the maintenance request entity, which is not shown in the figure, has many needed properties. It must include the location, the timestamp, the deadline, the severity level withthe impact of 
different types of faults, the particular device under repair, and the current status.The user applications that support the work responsibilities are designed to utilize the database for getting the correct information about the particular site/instance and update the data with the latest values based on the NOC operation. Of course, the applications must generate the required reports for the NOC engineers and the managers with the corresponding Key Performance Indicators (KPI).

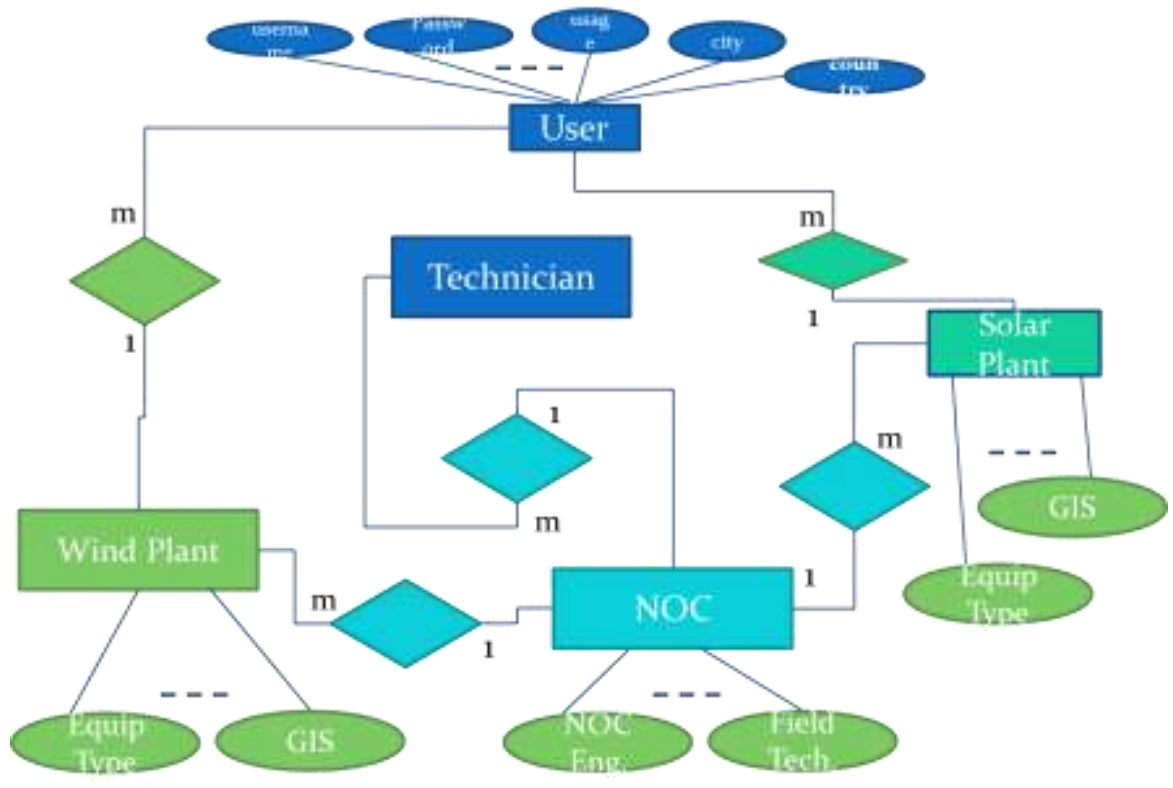

Fig. 5NOC database ERD - a simplified version

It is important to note that the NOC implementation can be done in a hierarchical manner. That is to mean that energy sources are divided into groups representing cities, regions, or countries. As an example, the renewable energy sources in a city can report to local NOC within the city which in turns, connects and reports to a main NOC in the country.

\section{Immersive NOC Environment}

Immersive environments are virtual reality-like, adding 3D abilities to present an ease of use with visualization abilities, to facilitate different capabilities:

- Visual simulation of the NOC environment.

- Gear the environment capabilities for education and awareness of energy purposes.

- Simulate energy scenarios at the consumption end.

- Visualize the energy source site structure.

- Simulate the operational maintenance scenarios.

- Visualize the preventive maintenance procedures.

- Promote the sense of presence at the production site.

Most of the work on immersive environments assumed usage in isolation of feeding sources. For example it is used for gaming, education, or simulation of real-life scenarios. However, it is believed that linking the information source, through the NOC infrastructure; to an immersive environment target the following goals: First, add the immersion abilities to the operation center. Second, act as a decision support tooland a part of smart city concept. Third, capture operational data and simulate operational what-if scenarios.Fourth, act as a tool to support the education and awareness aspects.

\section{Immersive NOC Operational Scenarios}

This section details some operational scenarios and show how the immersive environment can add value to the NOC team and the technical work environment. It is believed that building on this set, other relevant scenarios can be adopted and simulated through the NOC. In all the scenarios, it is evident that immersive environments add context through the 3D visual simulation and provide visual collaboration tools to remotely support decision making in the smart grid operation. 


\section{A. Teleportation to Production Site}

The field engineer has the ability to remotely use the immersive environment to be able to view the physical environment before actually dispatching to the site. Two modes of using the environment are expected. In a simulation mode, the field engineer dispatches virtually to the site. He can manipulate a virtual simulation of the device on site while under supervision and support. In this case, the user is able to assess the site in addition to be able to discover location specific details, such as areas under potential risk, the types of equipment available, and obtain the feel of location before physical dispatching to the site. Open Wonderland [12],as an example of immersive environment implementation, provides a one-click teleportation ability to locations. When the field engineer requires various types of support, the teleportation allows mixed existence with the remote team at NOC center with various means of visual collaborations based on the equipment 3D object model.With this scenario, GIS integration to the immersive environment should support this virtual teleportation to provide location specific support. Figure 6 illustrates an example of teleportation to a particular GISsite.

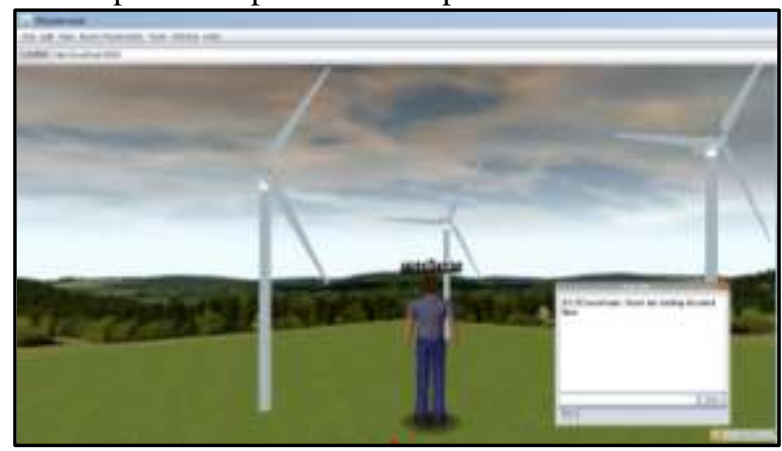

Fig. 6Example of teleportation to a particular GISsite

\section{B. Reseting a Particular Equipment}

Resetting particular equipment, or going into shutdown procedures, is expected and hence requires support. With the expected heterogeneity in a large scale, close monitoring and supervision of the task is required. Doing the task in a simulation environment provides an in-advance procedure that does not take actual effect but gives the pre-scenario to the field engineer or technician who performs the task; rather than the impact of shutting down a device or resetting an equipment and the corresponding consequences on the grid. When the technician or field engineer is dispatched to the site, the visual collaborative support offers detailed and synchronized instructions from the NOC location to the actual sites. Conversely, the actual status is applied to the environment and to the database, remotely, to reflect the status to all users.

\section{Preventive Maintenance Procedure}

In addition to the impact of a shutdown, maintenance operations should follow standardized and devicedependent procedures. Documentation about the steps needed should be available. Adding the 3D immersion abilities, rather than 2D sheets, giving the feel of context, should contribute to task completion on time. Remote visual collaboration supports the team on site through visualizing the procedures and providing directions through the communication channels of voice and text. An actual visual renderof the performed steps and the visual results are dependent on the ability to feedback actual scenes to the immersive environment.However, considering the traditional case of the immersive environment, it has been successful in providing training for such maintenance procedures. When the operation is complete, an update is fed back to the system to reflect on the grid/device status that is available to all users through the immersive environment.

\section{Assessing a Damaged Site}

Part of the grid monitoring activities is to assess damaged sites in order to take the appropriate procedures of bringing it back to production. A 3D map of the sites of production is recognized and used to traverse virtually to a site of interest. On the one end, there are the operation personnel who are remotely located from that site, but they need as much supportinginformation as possible. On the other end, there are the field personnel who are dispersed into the site. An increased support forthis new type communication between the site and the control center is needed, as well as among the personnel in site. Traditional voice and text are augmented with the $3 \mathrm{D}$ relevant models and database. Field personnel consequently update the model so that the status is shared among all users.

\section{E. Education and Awareness}

In order to promote renewable energy, the consumer can play a major role in the usability of the smart grid. In early stages of relying on renewable energy, the consumer is a very important player taking into 
consideration the economic factor. Optimizing the consumption and awareness by the consumer are important factors that are currently considered. Once the grid is available in the immersive environment, it is safe for users to be able to obtain a visualization of the energy production sites and devices. Students are able to traverse a wind turbine and obtain operational parameters for study reasons. Also, users of the grid should also be able to virtually visit those sites of production. Both of these increase the awareness of how energy is being produced. A web interface enables user access, with limited abilities, and instantaneous information of the grid status which in turn should reflect on their energy consumption behavior. It could be reinforced if there could be a chance for the consumer to observe some operational scenarios.

Research in immersive environments found an evidence on the impact of the environment on changing the user behavior after immersion [13]. However, increased support for massive population cannot rely on individual one-to-one support. Autonomous and 24/7 education support in immersive environments can provide a step ahead. The work in intelligent pedagogical agents can provide an intelligent non-conventional interface that is aided by pedagogical intelligence methods to support such awareness and answer specific questions about the NOC and the production environment [14,15]. The same work obtains an individual assessment to increase energy-awareness of citizens. Those citizens, especially with the new generation, are becoming more interested in utilizing new technologies to obtain better quality of life. For the younger generation, interacting with avatars in game like environmentsis more interesting than watching a complex dashboard.

\section{Conclusion}

The use of ENOC to monitor and control renewable energy sources is of great importance. Leaving the growth of these new energy resources to an ad-hoc situation with no proper planning will result in loss of power generation and distribution. The tracking of these scattered energy sources provides a comprehensive view of the availability of the energy sources and helps in the efficient usability of the produced energy. In this paper, we proposed an ENOC concept that provides IT support for the new energy sources. First, a comprehensive database of all the vital information of each energy site is designed. Second, the infrastructure of the ENOC is proposed with all the requirements needed to support the monitoring of the energy sites remotely. Third, an immersive ENOC user environment is proposed where a technician can receive 3D support in order to get his tasks performed. This 3D environment can be used in training the ENOC staff on the new operational scenarios.

The results of combining the technologies behind the ENOC and linking it to the immersive environment showed the added value of the $3 \mathrm{D}$ visualization in determining the best course of action on the operational scenarios of the system. The example scenarios presented here are basically used to illustrate the concepts introduced in the paper. For proper ENOC operation, a comprehensive list of scenarios that apply to the particular energy producing environment need to be developed and implemented. Moreover, the ENOC concept presented here can be turned into a business case for a particular city, region, or country. The expansion of these ideas is being considered in our future work.

\section{References}

[1] J. C.Foreman, R.K. Ragade, J. H. Graham; An Immersive Visualization Tool for Teaching and Simulation of Smart Grid Technologies; CoRR abs/1509.06293, 2015

[2] Continuum Managed Services Transformed; Retreived from http://www.continuum.net/msp-resources/mspedia/what-is-a-networkoperations-center-noc

[3] ManageEngine - Managed Service Provider; Retreived from https://www.manageengine.com/products/support-center

[4] NOC wall unit example; Retreived fromhttp://9xmedia.com/new/resources/applications.php

[5] Priya Mahadevan, Puneet Sharma, Sujata Banerjee, Parthasarathy Ranganathan; Energy Aware Network Operations; INFOCOM Workshops, IEEE, 2009

[6] Ameena Saad Al-Sumaiti, Mohammed Hassan Ahmed, Magdy M. A. Salama; Smart Home Activities: A Literature Review; Electric Power Components and Systems, Vol. 42, No. 3-4, 2014

[7] Rbee Solar; Retreived from http://rbeesolar.com

[8] Bimal Aklesh Kumar; Solar Power Systems Web Monitoring; The 2nd Symposium on Renewable Energy Technologies (SoRET), October 2011, Raiwai, Fiji

[9] Netbiter remote monitoring of solar power generators; Retreived from http://www.netbiter.com

[10] Phillip Homann, Fareed Ismail; Solar Park Plant Controller Design and Implementation within a South African Context; Retreived from http://www.concogrp.com

[11] Jacob Aho, Paul Fleming, et. al; Tutorial of Wind Turbine Control for Supporting Grid Frequency through Active Power Control; American Control Conference, Montreal, Canada June 27-29, 2012

[12] OpenWonderland, http://openwonderland.org/, Online, Accessed Jan. 20, 2015.

[13] S. J. Ahn, J. Bailenson; Embodied Experiences in Immersive Virtual Environments: Effects on Pro-environmental Self-efficacy and Behavior. $62^{\text {nd }}$ Annual International Communication Association Conference, May 24-28 2012, Phoenix, AZ

[14] M. Soliman, C. Guetl; Implementing Intelligent Pedagogical Agents in Virtual Worlds: Tutoring Natural Science Experiments in Open Wonderland; IEEE EDUCON 2013, Berlin, Germany

[15] M. Soliman, C. Guetl; Intelligent Pedagogical Agents in Immersive Virtual Learning Environments: A Review; Computers in Education, CE, MIPRO 2010, 2010, Croatia 